\title{
Lyapunov stability of flowing MHD plasmas surrounded by resistive walls
}

\author{
H. Tasso, G. N. Throumoulopoulos ${ }^{\dagger}$ \\ * Max-Planck-Institut für Plasmaphysik \\ Euratom Association \\ 85748 Garching bei München, Germany \\ $\dagger$ University of Ioannina, \\ Association Euratom-Hellenic Republic, \\ Department of Physics, GR 45110 Ioannina, Greece \\ September 9, 2018
}

*het@ipp.mpg.de

†gthroum@uoi.gr 


\begin{abstract}
A general stability condition for plasma-vacuum systems with resistive walls is derived by using the Frieman Rotenberg lagrangian stability formulation [Rev. Mod. Phys. 32, 898 (1960)]. It is shown that the Lyapunov stability limit for external modes does not depend upon the gyroscopic term but upon the sign of the perturbed potential energy only. In the absence of dissipation in the plasma such as viscosity, it is expected that the flow cannot stabilize the system.
\end{abstract}

PACS: 52.30.Jb, 52.35.Py, 02.90.+p 


\section{Introduction}

In a previous theorem [1] a general instability statement was proved concerning static plasmas surrounded by resistive walls. This statement is related to the problem of resistive wall modes (RWM) in the magnetohydrodynamic (MHD)literature. In particular, the impact of plasma rotation on the mode has been investigated theoretically in Refs. [2]-8] and experimentally in Refs.[9]-[11]. See also Ref.[12] for mode calculations in a cylindrical case and the recent review paper of Ref.[13]. In the present paper we show that the general Lyapunov analysis can be extended to stationary plasmas surrounded by resistive walls even if their resistivity is time-dependent as investigated in Ref.[14].

In section II we discuss the Lagrangian stability investigation of Ref. [15] in the context of free boundary displacements through an interface between plasma and vacuum. The Lyapunov functional is constructed in section III while conclusions and discussions are left to section IV.

\section{Stability equation}

The linearized Lagrangian displacement vector $\boldsymbol{\xi}$ about an ideal MHD equilibrium with flow obeys the equations derived in Ref.[15]:

$$
\rho_{0} \ddot{\boldsymbol{\xi}}+2 \rho_{0} \mathbf{v}_{\mathbf{0}} \cdot \nabla \dot{\boldsymbol{\xi}}+\mathbf{F} \boldsymbol{\xi}=0,
$$

where $\rho_{0}$ is the equilibrium mass density and $\mathbf{v}_{\mathbf{0}}$ the flow velocity. The antisymmetric $2 \rho_{0} \mathbf{v}_{\mathbf{0}} \cdot \nabla$ operator is sometime called the "gyroscopic" operator and $\mathbf{F}$ is given by

$\mathbf{F} \boldsymbol{\xi}=\nabla\left(\gamma p_{0} \nabla \cdot \boldsymbol{\xi}+\boldsymbol{\xi} \cdot \nabla p_{0}-\mathbf{B} \cdot \mathbf{Q}\right)+\mathbf{B} \cdot \nabla \mathbf{Q}+\mathbf{Q} \cdot \nabla \mathbf{B}+\nabla \cdot\left(\rho_{0} \boldsymbol{\xi} \mathbf{v}_{\mathbf{0}} \cdot \nabla \mathbf{v}_{\mathbf{0}}-\rho_{0} \mathbf{v}_{\mathbf{0}} \mathbf{v}_{\mathbf{0}} \cdot \nabla \boldsymbol{\xi}\right)$

where $p_{0}$ is the equilibrium pressure and $\mathbf{B}$ the equilibrium magnetic field. $\mathbf{Q}=\nabla \times(\boldsymbol{\xi} \times \mathbf{B})$ is the perturbed magnetic field. The operator $\mathbf{F}$ of Eq.(2) contains in particular flow terms so generalizing the corresponding operator of the static case [16].

After fixing the gauge by annihilating the scalar potential, the vector potential $\mathbf{A}$ in the region outside the plasma is governed by Ohm's law

$$
\nabla \times \nabla \times \mathbf{A}=-\sigma \dot{\mathbf{A}}
$$


where $\sigma$ is the space and time dependent conductivity in the outer region. $\sigma$ vanishes for a true vacuum.

At the interface between the plasma and the outer region, we must fulfil the condition

$$
\mathbf{n} \times \mathbf{A}=-(\mathbf{n} \cdot \boldsymbol{\xi}) \mathbf{B}_{\mathbf{0}}
$$

where $\mathbf{B}_{\mathbf{0}}$ is the equilibrium magnetic field at the vacuum side of the interface whose normal vector is $\mathbf{n}$. A has to vanish at infinity or be normal to superconducting walls, if there are any. Equations (1) to (4) reduce to the equations (1) to (3) of Ref.[14] if the flow vanishes.

The gyroscopic operator $2 \rho_{0} \mathbf{v}_{\mathbf{0}} \cdot \nabla$ is antisymmetric and the scalar product $2 \int \dot{\boldsymbol{\xi}} \cdot\left(\rho_{0} \mathbf{v}_{\mathbf{0}} \cdot \nabla\right) \dot{\boldsymbol{\xi}} d \tau$ as a volume integral vanishes for $\mathbf{n} \cdot \boldsymbol{\xi}=0$ at the plasma boundary. If $\mathbf{n} \cdot \boldsymbol{\xi} \neq 0$ at the interface the scalar product vanishes also if the mass density vanishes at the boundary. To make sure that this is true we use

$$
\begin{gathered}
\nabla \times\left(\boldsymbol{\xi} \times \rho_{0} \mathbf{v}_{\mathbf{0}}\right)=\rho_{0} \mathbf{v}_{\mathbf{0}} \cdot \nabla \boldsymbol{\xi}-\boldsymbol{\xi} \cdot \nabla\left(\rho_{0} \mathbf{v}_{\mathbf{0}}\right)-\rho_{0} \mathbf{v}_{\mathbf{0}} \nabla \cdot \boldsymbol{\xi} \\
\nabla\left(\boldsymbol{\xi} \cdot \rho_{0} \mathbf{v}_{\mathbf{0}}\right)=\rho_{0} \mathbf{v}_{\mathbf{0}} \cdot \nabla \boldsymbol{\xi}+\rho_{0} \mathbf{v}_{\mathbf{0}} \times \nabla \times \boldsymbol{\xi}+\boldsymbol{\xi} \cdot \nabla \rho_{0} \mathbf{v}_{\mathbf{0}}+\boldsymbol{\xi} \times \nabla \times \rho_{0} \mathbf{v}_{\mathbf{0}} .
\end{gathered}
$$

Note that the term $\boldsymbol{\xi} \nabla \cdot\left(\rho_{0} \mathbf{v}_{\mathbf{0}}\right)$ has vanishing contribution to Eq.(5) because of the equilibrium continuity equation. Adding equations (5) and (6) we obtain

$\nabla\left(\boldsymbol{\xi} \cdot \rho_{0} \mathbf{v}_{\mathbf{0}}\right)=2 \rho_{0} \mathbf{v}_{\mathbf{0}} \cdot \nabla \boldsymbol{\xi}-\rho_{0} \mathbf{v}_{\mathbf{0}} \nabla \cdot \boldsymbol{\xi}-\nabla \times\left(\boldsymbol{\xi} \times \rho_{0} \mathbf{v}_{\mathbf{0}}\right)+\rho_{0} \mathbf{v}_{\mathbf{0}} \times \nabla \times \boldsymbol{\xi}+\boldsymbol{\xi} \times \nabla \times \rho_{0} \mathbf{v}_{\mathbf{0}}$.

After taking the scalar product of Eq.(7) with $\boldsymbol{\eta}$ and using

$$
\begin{gathered}
\nabla \cdot\left[\boldsymbol{\eta} \times\left(\boldsymbol{\xi} \times \rho_{0} \mathbf{v}_{\mathbf{0}}\right)\right]=\left(\boldsymbol{\xi} \times \rho_{0} \mathbf{v}_{\mathbf{0}}\right) \cdot \nabla \times \boldsymbol{\eta}-\boldsymbol{\eta} \cdot \nabla \times\left(\boldsymbol{\xi} \times \rho_{0} \mathbf{v}_{\mathbf{0}}\right), \\
\nabla \cdot\left[\left(\boldsymbol{\eta} \cdot \rho_{0} \mathbf{v}_{\mathbf{0}}\right) \boldsymbol{\xi}\right]=\left(\boldsymbol{\eta} \cdot \rho_{0} \mathbf{v}_{\mathbf{0}}\right) \nabla \cdot \boldsymbol{\xi}+\boldsymbol{\xi} \cdot \nabla\left(\boldsymbol{\eta} \cdot \rho_{0} \mathbf{v}_{\mathbf{0}}\right),
\end{gathered}
$$

we integrate over the plasma volume and transform the volume integrals of the divergences to surface integrals at the interface to obtain

$$
\int_{V} \boldsymbol{\eta} \cdot\left(\rho_{0} \mathbf{v}_{\mathbf{0}} \cdot \nabla\right) \boldsymbol{\xi} d \tau=-\int_{V} \boldsymbol{\xi} \cdot\left(\rho_{0} \mathbf{v}_{\mathbf{0}} \cdot \nabla\right) \boldsymbol{\eta} d \tau
$$

if either $\mathbf{n} \cdot \boldsymbol{\xi}=\mathbf{n} \cdot \boldsymbol{\eta}=0$ for internal modes or $\rho_{0} \mathbf{v}_{\mathbf{0}}=0$ at the interface for external modes. 


\section{Lyapunov functional}

Taking the scalar product of Eq.(1) with $\dot{\boldsymbol{\xi}}$ and Eq.(3) with $\dot{\mathbf{A}}$ and integrating over the plasma volume and the vacuum region respectively we obtain

$$
\frac{d(K+W)}{d t}=-(\sigma \dot{\mathbf{A}}, \dot{\mathbf{A}})
$$

where

$$
\begin{array}{r}
W=\frac{(\boldsymbol{\xi}, \mathbf{F} \boldsymbol{\xi})_{p}+(\mathbf{A}, \nabla \times \nabla \times \mathbf{A})_{o r}}{2}, \\
K=\frac{\left(\rho_{0} \dot{\boldsymbol{\xi}}, \dot{\boldsymbol{\xi}}\right)_{p}}{2},
\end{array}
$$

the subscripts $p$ and or being related to the plasma and the outer region respectively. The parentheses denote the scalar products

$$
\begin{aligned}
& (\boldsymbol{\eta}, \boldsymbol{\mu})_{p}=\int_{p}(\boldsymbol{\eta}, \boldsymbol{\mu}) d \tau \\
& (\boldsymbol{\eta}, \boldsymbol{\mu})_{o r}=\int_{o r}(\boldsymbol{\eta}, \boldsymbol{\mu}) \tau .
\end{aligned}
$$

Since $\sigma$ is positive, the quantity $K+W$ has a negative time derivative for external modes according to Eq.(11). This allows us to apply the method of Lyapunov, which states that if $K+W$ has no definite sign, then the system is "Lyapunov unstable". For internal modes with $\mathbf{A}=0$ the condition is only sufficient. The Hermitian form $W$ could be minimized in the same way as the $\delta W$ of the energy principle [16] for static MHD equilibria, but no numerical code for minimizing $W$ has been developed yet. For a straight tokamak with homogeneous axial flow and without walls $W$ is indefinite if it is indefinite in the static case. This follows from the fact that the stability property does not depend upon the inertial frame chosen. Therefore, according to the above Lyapunov study the introduction of resistive walls cannot stabilize the plasma-wall system with finite constant plasma velocity. One should note, however, that Lyapunov stability is equivalent to spectral stability if all modes of the spectrum are considered. This is not in agreement of the result of Ref.[6]. In general, the entire spectrum of the plasma-wall system is not as easy to investigate as the determination of the sign of $W$.

Eq.(11) reduces to Eq.(4) of Ref.[1, 14] if the velocity terms in $F$ vanish. It extends the Lyapunov functional introduced in Ref.[1, 14] to the present 
general flow case. This means that if $W$ is positive definite the system is stable, but if it is indefinite the system is unstable. So the gyroscopic operator does not affect stability of external modes but growth rates, eigenvalues and eigenfunctions only.

\section{Discussion and conclusions}

The question of stability of external modes for toroidal ideal plasmas with flows surrounded by resistive walls is decided by the sign of the symmetric potential operator only, which is a drastic simplification so that numerical investigation of stability becomes only slightly more difficult than in the static case for which many codes have been developed.

Moreover the Lyapunov stability property holds also for internal modes if a dissipation operator is added to the gyroscopic operator in (1) since this addition would not change the sign of the right hand side of (11). However, if we add a viscosity to the physical system under investigation then (1) would be modified by such an operator acting on $\dot{\xi}$ but also by another operator acting on $\boldsymbol{\xi}$ which represents the "circulatory" forces as considered in Ref.[17]. The operator on $\dot{\xi}$ is proportional to the viscosity itself but the "circulatory" operator goes with viscosity times the velocity $\mathbf{v}_{\mathbf{0}}$. Only the latter operator can modify the stability property, so a minimum velocity is needed for this operator to be able to stabilize the system. However, the quantitative investigation of such situations is difficult as explained in Ref.[17] and Ref.[18. We should also mention that the plasma viscosity is a tensor known only in the collisional regime while a convincing derivation for the weak collisionality regime of fusion plasmas has not been done yet. Therefore, it may turn out that the stability of hot plasmas must be treated by kinetic equations like Vlasov or Fokker-Planck for which the present stability procedure does not apply.

Finally, if for physical interpretations of the experiment, eigenmodes and growth rates of (1) to (4) (and possibly nonlinear effects) are needed, then the investigation of such situations will be much more difficult especially for toroidal plasmas. Numerical techniques for the search of complex eigenvalues must be developed in the first place. (See e.g. [19]). 


\section{Acknowledgements}

Part of this work was conducted during a visit of one of the authors (G.N.T.) to the Max-Planck-Institut für Plasmaphysik, Garching. The hospitality of that Institute is greatly appreciated. The present work was performed under the Contract of Association ERB 5005 CT 990100 between the European Atomic Energy Community and the Hellenic Republic.The views and opinions expressed herein do not necessarily reflect those of the European Commission. 


\section{References}

[1] D. Pfirsch, H. Tasso, Nuclear Fusion 11, 259 (1971).

[2] A. Bondeson and D.J. Ward, Pys. Rev. 72, 2709 (1994).

[3] C.N. Lashmore-Davies, Phys. Plasmas 8, 151 (2001).

[4] M.S. Chu, V.S. Chan, M.S. Chance et al. Nucl. Fusion 43, 196 (2003).

[5] M. Sato, S. Hamaguchi, and M. Wakatani, Phys. Plasmas 10, 187 (2003).

[6] S.P. Smith, S.C. Jardin, J.P. Freidberg, and L. Guazzoto, Physics of Plasmas 16, 084504 (2009).

[7] L.J. Zheng, M.T. Kotschenreuther, and J.W. Van Dam, Physics of Plasmas 17, 056104 (2010).

[8] C.J. Ham, C.G. Gimblett and R.J. Hastie, Nuclear Fusion 53, 025001 (2011).

[9] H. Reimerdes, A.M. Garofalo, G.L. Jackson et al. Phys. Rev. Lett. 98, 055001 (2007).

[10] G. Matsunaga, N. Aiba, K. Shinohara et al., Phys. Rev. Lett. 103, 045001 (2009).

[11] J.W. Berkery, S.A. Sabbagh, R. Betti et al., Phys. Rev. Let. 104, 035003 (2010).

[12] J.P. Goedbloed, D. Pfirsch and H. Tasso, Nuclear Fusion 12, 649 (1972).

[13] M.S. Chu and M. Okabayashi, Plasma Phys. Control. Fusion 52, 1 (2010).

[14] H. Tasso and G.N. Throumoulopoulos, Physics of Plasmas 11, 334 (2004).

[15] E. Frieman and M. Rotenberg, Rev. Mod. Phys. 32, 898 (1960).

[16] I. Bernstein, E.A. Frieman, M.D. Kruskal and R.M. Kulsrud, Proc. Roy. Soc. (London) A244, 17 (1958). 
[17] H. Tasso, ZAMP 31, 536 (1980).

[18] H. Tasso and G.N. Throumoulopoulos, Phys. Lett. A 271, 413 (2000).

[19] W. Kerner, Journal Comput. Phys. 85, 1 (1989). 